\title{
Catechol O-Methyltransferase Haplotype Predicts Immediate Musculoskeletal Neck Pain and Psychological Symptoms after Motor Vehicle Collision
}

\author{
Samuel A. McLean1 1,2,3, Luda Diatchenko ${ }^{2}$, Young M. Lee ${ }^{1,2}$, Robert A. Swor ${ }^{4}$, Robert M. \\ Domeier $^{5}$, Jeffrey S. Jones ${ }^{6}$, Christopher W Jones ${ }^{1,3}$, Caroline Reed ${ }^{1}$, Richard E. Harris ${ }^{5}$, \\ William Maixner ${ }^{2}$, Daniel J. Clauw ${ }^{5}$, and Israel Liberzon ${ }^{9}$ \\ ${ }^{1}$ TRYUMPH Research Program (SAM, YML, CWJ, CR), University of North Carolina, Chapel Hill, \\ NC \\ ${ }^{2}$ Center for Neurosensory Disorders (SAM, LD, WM, YML), University of North Carolina, Chapel \\ Hill, NC \\ ${ }^{3}$ Department of Emergency Medicine (SAM, CWJ), University of North Carolina, Chapel Hill, NC \\ ${ }^{4}$ Department of Emergency Medicine, William Beaumont Hospital, Royal Oak, MI (RAS) \\ ${ }^{5}$ Department of Emergency Medicine, St Joseph Mercy Hospital, Ann Arbor, MI (RMD) \\ ${ }^{6}$ Department of Emergency Medicine, Spectrum Health Hospital - Butterworth Campus, Grand \\ Rapids, MI (JSJ) \\ ${ }^{5}$ Department of Anesthesiology (REH, DJC), University of Michigan, Ann Arbor, MI \\ ${ }^{9}$ Department of Psychiatry (IL), University of Michigan, Ann Arbor, MI
}

\begin{abstract}
Genetic variations in the catechol-o-methyltransferase (COMT) gene have been associated with experimental pain and risk of chronic pain development, but no studies have examined genetic predictors of neck pain intensity and other patient characteristics after motor vehicle collision (MVC). We evaluated the association between COMT genotype and acute neck pain intensity and other patient characteristics in 89 Caucasian individuals presenting to the emergency department (ED) after MVC. In the ED in the hours after MVC, individuals with a COMT pain vulnerable genotype were more likely to report moderate to severe musculoskeletal neck pain ( $76 \%$ vs. $41 \%$,
\end{abstract}

(C) 2010 The American Pain Society. Published by Elsevier Inc. All rights reserved.

Manuscript correspondence: Samuel McLean, MD, TRYUMPH Research Program, University of North Carolina, 2100 Old Dental Building, CB\#7455, Chapel Hill, NC 27599-7455 Address for Reprints: Same Phone: 919-966-7315 Fax: 919-843-9812 samclean@email.unc.edu.

Publisher's Disclaimer: This is a PDF file of an unedited manuscript that has been accepted for publication. As a service to our customers we are providing this early version of the manuscript. The manuscript will undergo copyediting, typesetting, and review of the resulting proof before it is published in its final citable form. Please note that during the production process errors may be discovered which could affect the content, and all legal disclaimers that apply to the journal pertain.

Subject Headings: Musculoskeletal pain, Catechol O-methyltransferase, motor vehicle collision, pain, stress, WAD

Scientific Meeting Presentation: McLean SA, Diatchenko L, Reed C, Jones CW, Zaleski E, Mistry Y, Swor R, Sochor MR, Liberzon I, Clauw D, Maixner W. Catechol $O$-Methyltransferase (COMT) met/met genotype influences cortisol response and pain symptoms after minor motor vehicle collision (MVC). Presented at the Annual Meeting of the American Pain Society, Tampa Bay, FL, 2008. J Pain 9(4):8, 2008.

Perspective: The association of COMT genotype with pain symptoms, psychological symptoms, and recovery beliefs exemplifies the pleiotropic effects of stress-related genes which may provide the biological substrate for the biopsychosocial model of post-MVC pain. The identification of genes associated with post-MVC symptoms may also provide new insights into pathophysiology. 
$\mathrm{RR}=2.11(1.33-3.37))$, moderate or severe headache $(61 \%$ vs. $33 \%, \mathrm{RR}=3.15(1.05-9.42)$, and moderate or severe dizziness ( $26 \%$ vs. $12 \%, \mathrm{RR}=1.97(1.19-3.21)$ ). Individuals with a pain vulnerable genotype also experienced more dissociative symptoms in the ED, and estimated a longer time to physical recovery (median 14 vs. 7 days, $p=.002$ ) and emotional recovery (median 8.5 vs. 7 days, $\mathrm{p}=.038$ ). These findings suggest that genetic variations affecting stress response system function influence the somatic and psychological response to MVC, and provide the first evidence of genetic risk for clinical symptoms after MVC.

\section{Introduction}

Musculoskeletal neck pain development after motor vehicle collision (MVC) is an international public health problem. ${ }^{5}$ Consistent with the term "Whiplash Associated Disorders", biologic mechanisms influencing acute post-MVC neck pain have largely been conceptualized as cervical sprain/strain during the MVC event. Such soft tissue damage is difficult to directly assess, and therefore crash characteristics (e.g. direction of collision, severity of vehicle damage, presence/location of headrest) have been used as indirect measures of mechanical force experienced by neck tissues during the collision.2,5, 6 However, such crash characteristics are poor predictors of pain outcomes.5 As a result, biologic mechanisms generating post-MVC pain remain unsubstantiated, to the great frustration of both patients and caregivers.

In addition to exposing soft tissues to biomechanical stress, a MVC is also a potent stressor. ${ }^{19}$ Acute stressors activate the sympathetic nervous system and adrenomedullary hormonal system, resulting in the release of epinephrine and norepinephrine. ${ }^{10}$ Increasing evidence suggests that sympathetic activation may contribute to pain development via mechanisms both independent from and interactive with pain mechanisms related to tissue injury and inflammation. ${ }^{12,13,17}$ Such adrenergic mechanisms may influence the development of neck pain symptoms after MVC. If so, then genetic variations affecting the function of important adrenergic system components may contribute to individual variation in vulnerability to musculoskeletal pain symptoms after MVC.

One key component of the adrenergic system is the catechol $O$-methyltransferase (COMT) enzyme. COMT is the primary enzyme which degrades catecholamines, including epinephrine, norepinephrine, and dopamine. Variants of the COMT gene have been associated with experimental pain sensitivity ${ }^{7,27}$ and with vulnerability to both chronic pain $^{7,23}$ and anxiety disorders ${ }^{11,25}$. These observations provide a strong rationale for examining the association between genetic variants of COMT and clinical pain intensity and related symptoms following MVC.

In this study we assessed the association between COMT variation and the intensity of acute neck pain experienced in the emergency department (ED) during the hours after MVC. We selected this patient characteristic as our primary outcome measure because initial neck pain severity after MVC is one of the strongest predictors of chronic neck pain development. ${ }^{5} \mathrm{We}$ hypothesized that individuals with a COMT pain vulnerable genotype, who have relatively high catecholamine levels, would experience more intense post-MVC neck pain. We also assessed the association between COMT pain vulnerable genotype and ED distress and dissociation symptoms, other somatic symptoms (i.e. headache and dizziness), and patient cognitions about their recovery. 


\section{Methods}

\section{Participants and Inclusion Criteria}

Eighty nine individuals $\geq 18$ years of age presenting to three emergency departments (EDs) for care after MVC were recruited for the study. Only non-pregnant, non-admitted, Englishspeaking patients who were alert and oriented, stable, and without spinal, facial, or skull fracture, intraoral laceration, or intracranial injury were enrolled. Patients taking $\beta$ adrenergic antagonist medications were also excluded. Patients of Caucasian ancestry constitute the majority of local ED patients, thus to avoid genetic population stratification bias only Caucasian patients were included in this analysis. Institutional Review Board approval was obtained from study site IRBs (William Beaumont Hospital IRB, Royal Oak, MI, St Joseph Mercy Hospital IRB, Ann Arbor, MI, and Spectrum Health/Butterworth Hospital IRB, Grand Rapids, MI). All patients gave written informed consent.

\section{COMT Pain Vulnerable Genotype Definition}

When multiple disease susceptibility variants occur in the same gene, the overall functional state of the gene may not be easily deduced from information regarding a single SNP. ${ }^{16}$ For this reason we used a haplotype-based approach to examining COMT functional variants. In Caucasians, three major haplotypes located in the central COMT locus account for approximately $96 \%$ of all haplotypes and can be determined by the combination of single nucleotide polymorphisms present at rs6269, rs4633, rs4818, and rs4680. ${ }^{7}$ Based on their association with sensitivity to experimental pain stimuli, these three haplotypes have been termed the average pain sensitivity haplotype (A_T_C_A), the high pain sensitivity haplotype (A_C_C_G), and the low pain sensitivity (LPS) haplotype (G_C_G_G). ${ }^{7}$ The LPS haplotype produces much higher levels of COMT enzymatic activity than either the APS or HPS haplotypes and is associated with the lowest pain sensitivity.7 Based on these data, genotypes that did not include at least one copy of the LPS haplotype were defined a priori as COMT pain vulnerable genotypes.

\section{Blood Collection and Genotyping}

Blood was collected in the ED for subsequent DNA analysis using Paxgene DNA Collection tubes (PreAnalytiX, Hilden, Germany). DNA extraction and genotyping (at rs4633, rs4680, rs4818, and rs6269) were performed by Clinical Data, Inc. (Morrisville, NC) using the Sequenom platform. Genotype at these four SNPs was used to identify individuals with and without a pain vulnerable genotype.

\section{Participant Assessments}

In addition to blood sample collection, participant and crash characteristics assessed in the ED included the following:

ED Neck Pain Intensity-ED neck pain intensity among patients with and without a $C O M T$ pain vulnerable genotype was selected a priori as the primary study outcome. ED neck pain intensity was assessed using a verbal 0-10 numeric rating scale (NRS). Verbal scores have advantages in acute care settings, and verbally administered NRSs have been validated as a substitute for VASs in acute pain measurement in the ED.3 A previous study9 identified a score of 4 or 5 as the optimal lower limit for moderate or severe neck pain (based on interference) using a 0-10 NRS. A score of 4 was chosen as the lower limit for moderate pain as this is also consistent with clinical practice14. 
Intensity of Other Somatic Symptoms-Intensity of other somatic symptoms (i.e. headache and dizziness) were assessed using a verbal 0-10 numeric rating scale (NRS). A NRS score $>3$ was used to define moderate or severe symptom intensity.

Distress and Dissociative Symptoms-The Peritraumatic Distress Inventory4 is a 13item $(0-4$ Likert scale response for each item, score range 0 - 52) self-report measure which provides a quantitative measure of the level of distress experienced during and immediately after a traumatic event. The Michigan Critical Events Perception Scale ${ }^{15}$ (MCEPS) is a 5-item scale ( $1-5$ Likert scale, score range 5-25) which provides a quantitative assessment of the level of peritraumatic dissociation. A mean MCEPS score > 3 defined significant dissociation. 15

Expectations of Recovery-Participants were asked to estimate their likelihood of recovery on a $0-100 \%$ scale. Participants were also asked to provide estimates of the amount of time it would take them to recover physically and emotionally from the MVC; all answers were converted to days.

Injury Assessment-Injury information was obtained from the medical record. Injuries were classified according to whether they included a laceration or small bone fracture. Individuals without a laceration, fracture, or other identifiable tissue pathology were defined as having musculoskeletal strain only. Injury information was also used to calculate Abbreviated Injury Scale (AIS) score ${ }^{1}$. AIS score is an anatomical scoring system which codes injuries according to anatomic location and type and assigns a severity score to each injury based on this information. Severity scores range from 1-6, a score of 1 denotes minor severity and a score of 2 denotes moderate severity.

Demographic and Crash Assessment-Demographic and crash characteristics (car drivable from scene, rear end collision, airbag deployment) were assessed via patient interview. Information regarding the time of the MVC and the time of ED presentation were abstracted from the medical record.

\section{Statistical Analysis}

ED neck pain intensity among patients with and without a COMT pain vulnerable genotype was selected a priori as the primary study outcome. Prior to analyses, variable distributions were assessed for normality via histogram and Kolmogorov-Smirnov statistic. ED neck pain intensity (primary outcome variable), intensity of headache and dizziness symptoms, and participant cognitive characteristics were not normally distributed. Therefore these characteristics were assessed as categorical variables (using cut-offs described above) via relative risk with 95\% confidence interval or Fisher's Exact Test. Neck pain intensity levels among those with and without a COMT pain vulnerable genotype were also assessed using median values and Mann-Whitney $U$ test, as were cognitive characteristics. Psychological symptoms (ED distress and dissociative symptoms) were normally distributed, therefore bivariate analyses of psychological symptoms utilized mean values and student's $t$ test.

Forward stepwise regression models ( $\mathrm{p}$ in $=.1$, $\mathrm{p}$ out $=.15$ ) were also used to identify the predictor(s) most strongly associated with acute pain and psychological symptoms.

Somewhat liberal $\mathrm{p}$ value criteria were chosen due to our relatively modest sample size. For each model, candidate independent variables included demographic characteristics (age, gender, income, education), crash and injury characteristics (highest AIS score, whether car was drivable at scene, and whether airbags deployed), and presence or absence of COMT pain vulnerable genotype. Logistic regression (likelihood ratio) models were used for categorical variables, and linear regression models for continuous variables. All regression 
models were evaluated for assumptions and aptness. Statistical analyses were performed using SPSS software (SPSS Inc., Chicago, IL).

\section{Results COMT Haplotypes}

Of eighty nine genotyped enrollees, 4 (4.5\%) individuals had rare COMT haplotypes. The effect of these rare haplotypes on COMT enzyme activity and experimental pain sensitivity is not known, therefore these individuals were excluded from analyses. Participant demographic and injury characteristics are shown in Table 1. Consistent with previously reported Caucasian haplotype frequencies, ${ }^{7} 40 \%$ of participants had no LPS allele and were classified as having a COMT pain vulnerable genotype.

\section{Patients}

Most participants had at least some post-secondary school education and worked full time (Table 1). Median income category among participants was $\$ 40,000$ - $\$ 60,000$. More than $90 \%$ of participants had musculoskeletal strain only, and most arrived in the ED less than two hours after the MVC occurred. Twenty six participants (29\%) reported airbag deployment during the collision. Fifty two (58\%) came directly from the accident scene; 34 (59\%) of these were transported to the ED by ambulance. Slightly more than half of participants reported moderate or severe acute neck pain in the ED. Patient characteristics did not differ between study sites.

\section{Association of COMT Pain Vulnerable Genotype with Participant Characteristics}

Highest AIS score in those with and without a COMT pain vulnerable genotype were very similar (1.11 (.32) vs. $1.08(.28), \mathrm{p}=.62)$. The percentage of individuals with and without a COMT pain vulnerable genotype who reported that their car was drivable after the MVC was also very similar ( $43 \%$ vs. $40 \%, \mathrm{p}=.83$ ), as was the percentage of individuals in the two groups with musculoskeletal injury only (91\% vs. 90\%, p > .99). Patients with a COMT pain vulnerable genotype tended to come to the ED more often after experiencing a rear end collision ( $57 \%$ vs. $36 \%, \mathrm{p}=.08)$, and to not have had an airbag deploy during the collision $(17 \%$ vs. $39 \%, \mathrm{p}=.06)$.

\section{Association of COMT Pain Vulnerable Genotype with ED Neck Pain Intensity}

Table 2 displays the association between selected participant and crash characteristics and the presence or absence of moderate or severe neck pain symptoms in the ED after MVC. Relative risk point estimates suggest that younger age, female gender, low income, low education level, and rear end collision were associated with an increased risk of moderate or severe ED neck pain, and airbag deployment with reduced risk. However, as the confidence intervals show, none of these associations reached statistical significance in the present sample.

A significant association was observed between having a COMT pain vulnerable genotype and experiencing moderate or severe acute neck pain (Table 2). Patients with a COMT pain vulnerable genotype also had a significantly higher median neck pain score than patients without the COMT pain vulnerable genotype (6 vs. $2, \mathrm{U}=.001$ ). A forward stepwise logistic regression model using candidate demographic, crash, injury, and COMT pain vulnerable genotype characteristics selected income and COMT pain vulnerable genotype for model entry (Table 3). 


\section{Association of COMT Pain Vulnerable Genotype with other Somatic Symptoms}

A significant association was observed between having a COMT pain vulnerable genotype and moderate or severe headache $(61 \%$ vs. $33 \%, \mathrm{RR}=3.15(1.05-9.42))$ and moderate or severe dizziness ( $26 \%$ vs. $12 \%, \mathrm{RR}=1.97(1.19-3.21)$ ). Final forward stepwise logistic regression models using candidate demographic, crash, injury, and COMT pain vulnerable genotype characteristics selected COMT pain vulnerable genotype only for model entry (Table 3).

\section{Association of COMT Pain Vulnerable Genotype with Psychological Symptoms and Cognitions}

A significant association was observed between COMT pain vulnerable genotype and increased dissociative symptoms (mean MCEPS score 2.7 (1.0) vs. $2.2(.9), \mathrm{p}=.016$ ). The final forward stepwise logistic regression models selected COMT pain vulnerable genotype and rearend collision type for model entry (Table 4). In contrast, the association between COMT pain vulnerable genotype and increased distress symptoms did not reach significance (mean distress symptom score $19.8(10.3)$ vs. $17.4(9.1), \mathrm{p}=.284$ ). The final predictive model for dissociative symptoms selected income and airbag deployment (Table 4). In addition, patients with a COMT pain vulnerable genotype estimated a longer time to physical recovery (median 14 vs. 7 days, $p=.002$ ) and emotional recovery (median 8.5 vs. 7 days, $\mathrm{p}=.038)$, and tended to be less certain of full recovery $(\mathrm{p}=.055)$.

\section{Discussion}

COMT pain vulnerable genotype predicted acute somatic and psychological symptoms after MVC in our sample. Seventy six percent of individuals with a COMT pain vulnerable genotype reported moderate or severe neck pain in the ED in the hours after MVC, vs. $41 \%$ of those without a COMT pain vulnerable genotype. Individuals with a COMT pain vulnerable genotype also experienced more dissociative symptoms, were more likely to experience other somatic symptoms in the ED, and estimated a longer time to physical and emotional recovery. The presence or absence of a COMT pain vulnerable genotype was more predictive of moderate or severe acute neck pain than the crash-related characteristics assessed, including rear-end collision type, airbag deployment, and whether or not the car was drivable at the scene.

Patients with a COMT pain vulnerable genotype tended to be more likely to have experienced a rear end collision and to be less likely to have had an airbag deploy. These associations are linked, because motor vehicles struck from the rear are less likely to have an airbag deploy than those struck from the front or side. If rear end collisions are inherently more likely to result in acute neck pain, then the association between COMT genotype and pain and distress symptoms may be confounded by the association between COMT genotype and rear end collision. However, available data indicates that this is not the case. First, the association between COMT pain vulnerable genotype and moderate or severe ED neck pain remains when stratified by crash type (e.g. $\mathrm{p}=.028$ for association between ED neck pain and COMT pain vulnerable genotype among patients involved in a rear end collision only). Second, as shown in Table 2, the association between rear end collision and moderate or severe ED neck pain is itself modest. Finally, previous studies have found little association between impact direction and neck pain symptoms. ${ }^{5}$

Findings from other settings indicate that individuals with a COMT pain vulnerable genotype are at increased risk of chronic pain. ${ }^{7,23}$ Our findings suggest that individuals with a COMT pain vulnerable genotype experience more intense acute pain and somatic symptoms after a collision. Because of increased initial symptoms, individuals with a COMT 
pain vulnerable genotype may also be more likely to seek ED care after MVC. If so, such shunting of vulnerable individuals to emergency care settings may help create opportunities to identify high risk individuals and provide preventive interventions in the early aftermath of MVC.

The influence of the COMT pain vulnerable genotype on post-MVC pain symptoms, dissociation symptoms, beliefs regarding recovery, and perhaps the decision to seek care itself exemplifies the pleiotropic effects of the stress-related genes which provide the biological substrate for the biopsychosocial model of post-MVC pain. The identification of genes associated with vulnerability to post-MVC symptoms may provide new insights into potential preventive interventions for high risk individuals. Improved understanding of biological processes contributing to post-MVC symptom development may also reduce the stigma that is unfortunately still commonly experienced by individuals developing pain and psychological symptoms after MVC.

Several limitations should be considered when interpreting our study results. First, the present study only examined the influence of the COMT pain vulnerable genotype on patient symptoms in the ED. Further studies are necessary which assess both immediate and persistent pain and psychological outcomes after MVC. Second, our sample size was relatively small, and this small sample size likely resulted in a lack of significance for some of our associations. However, we were able to detect a number of the most robust findings regarding COMT haplotypes. Third, our secondary analyses were not adjusted for multiple comparisons. However, the need for such adjustment is debated, ${ }^{8,18,20-22}$ and to help address this issue we selected a single exposure (COMT pain vulnerable genotype) and a primary outcome (ED neck pain severity) in advance of the analysis ${ }^{8}, 18,26$. In addition, our assessments of potential confounders were relatively detailed, and the results of our secondary analyses (association of COMT genotype with somatic symptoms, anxiety symptoms, and cognitions) are consistent with the results of previous work. ${ }^{7,11,24-27}$ Larger cohort studies are needed to confirm an association between genetic variations in COMT and both immediate and persistent pain and psychological symptoms after MVC.

\section{Acknowledgments}

Funding for this study came from Dr. McLean's NIH K23 KAR050410A and institutional resources. In addition to their primary academic appointments, Drs. McLean, Diatchenko, and Maixner are corporate officers and share holders of Algynomics, Incorporated. Dr. Clauw reports past consulting relationships with Cypress Biosciences, Lilly, Wyeth, Pfizer, Johnson and Johnson, and Forest.

Funding: Funding for this study came from Dr. McLean's NIH K23 KAR050410A and institutional resources.

\section{References}

1. The Abbreviated Injury Scale (AIS) 1990 Revision. Association for the Advancement of Automotive Medicine; Des Plaines, IL: 1990.

2. Berglund A, Bodin L, Jensen I, Wiklund A, Alfredsson L. The influence of prognostic factors on neck pain intensity, disability, anxiety and depression over a 2-year period in subjects with acute whiplash injury. Pain 2006;125:244-256. [PubMed: 16806708]

3. Bijur PE, Latimer CT, Gallagher EJ. Validation of a verbally administered numerical rating scale of acute pain for use in the emergency department. Acad Emerg Med 2003;10:390-392. [PubMed: 12670856]

4. Brunet A, Weiss DS, Metzler TJ, Best SR, Neylan TC, Rogers C, Fagan J, Marmar CR. The Peritraumatic Distress Inventory: a proposed measure of PTSD criterion A2. American Journal of Psychiatry 2001;158:1480-1485. [PubMed: 11532735]

5. Carroll LJ, Holm LW, Hogg-Johnson S, Cote P, Cassidy JD, Haldeman S, Nordin M, Hurwitz EL, Carragee EJ, van der Velde G, Peloso PM, Guzman J. Course and prognostic factors for neck pain 
in whiplash-associated disorders (WAD): results of the Bone and Joint Decade 2000-2010 Task Force on Neck Pain and Its Associated Disorders. Spine (Phila Pa 1976) 2008;33:S83-92. [PubMed: 18204405]

6. Cassidy JD, Carroll LJ, Cote P, Lemstra M, Berglund A, Nygren A. Effect of eliminating compensation for pain and suffering on the outcome of insurance claims for whiplash injury. New England Journal of Medicine 2000;342:1179-1186. [PubMed: 10770984]

7. Diatchenko L, Slade GD, Nackley AG, Bhalang K, Sigurdsson A, Belfer I, Goldman D, Xu K, Shabalina SA, Shagin D, Max MB, Makarov SS, Maixner W. Genetic basis for individual variations in pain perception and the development of a chronic pain condition. Hum Mol Genet 2005;14:135143. [PubMed: 15537663]

8. Feise RJ. Do multiple outcome measures require p-value adjustment? BMC Med Res Methodol 2002;2:8. [PubMed: 12069695]

9. Fejer R, Jordan A, Hartvigsen J. Categorising the severity of neck pain: establishment of cut-points for use in clinical and epidemiological research. Pain 2005;119:176-182. [PubMed: 16298059]

10. Goldstein DS. Catecholamines and stress. Endocr Regul 2003;37:69-80. [PubMed: 12932192]

11. Hettema JM, An SS, Bukszar J, van den Oord EJ, Neale MC, Kendler KS, Chen X. Catechol-Omethyltransferase contributes to genetic susceptibility shared among anxiety spectrum phenotypes. Biol Psychiatry 2008;64:302-310. [PubMed: 18436194]

12. Khasar SG, Green PG, Miao FJ, Levine JD. Vagal modulation of nociception is mediated by adrenomedullary epinephrine in the rat. Eur J Neurosci 2003;17:909-915. [PubMed: 12603283]

13. Khasar SG, McCarter G, Levine JD. Epinephrine produces a beta-adrenergic receptor-mediated mechanical hyperalgesia and in vitro sensitization of rat nociceptors. J Neurophysiol 1999;81:1104-1112. [PubMed: 10085337]

14. Krebs EE, Carey TS, Weinberger M. Accuracy of the pain numeric rating scale as a screening test in primary care. J Gen Intern Med 2007;22:1453-1458. [PubMed: 17668269]

15. Michaels AJ, Michaels CE, Zimmerman MA, Smith JS, Moon CH, Peterson C. Posttraumatic stress disorder in injured adults: etiology by path analysis. Journal of Trauma-Injury Infection \& Critical Care 1999;47:867-873.

16. Nackley AG, Shabalina SA, Tchivileva IE, Satterfield K, Korchynskyi O, Makarov SS, Maixner W, Diatchenko L. Human catechol-O-methyltransferase haplotypes modulate protein expression by altering mRNA secondary structure. Science 2006;314:1930-1933. [PubMed: 17185601]

17. Nackley AG, Tan KS, Fecho K, Flood P, Diatchenko L, Maixner W. Catechol-O-methyltransferase inhibition increases pain sensitivity through activation of both beta(2)- and beta(3)-adrenergic receptors. Pain. 2006

18. Perneger TV. What's wrong with Bonferroni adjustments. BMJ 1998;316:1236-1238. [PubMed: 9553006]

19. Pervanidou P, Margeli A, Lazaropoulou C, Papassotiriou I, Chrousos GP. The immediate and longterm impact of physical and/or emotional stress from motor vehicle accidents on circulating stress hormones and adipo-cytokines in children and adolescents. Stress 2008;11:438-447. [PubMed: 19065457]

20. Rothman KJ. No adjustments are needed for multiple comparisons. Epidemiology 1990;1:43-46. [PubMed: 2081237]

21. Savitz DA, Olshan AF. Multiple comparisons and related issues in the interpretation of epidemiologic data. Am J Epidemiol 1995;142:904-908. [PubMed: 7572970]

22. Savitz DA, Olshan AF. Describing data requires no adjustment for multiple comparisons: a reply from Savitz and Olshan. Am J Epidemiol 1998;147:813-814. discussion 815. [PubMed: 9583710]

23. Vargas-Alarcon G, Fragoso JM, Cruz-Robles D, Vargas A, Lao-Villadoniga JI, Garcia-Fructuoso F, Ramos-Kuri M, Hernandez F, Springall R, Bojalil R, Vallejo M, Martinez-Lavin M. CatecholO-methyltransferase gene haplotypes in Mexican and Spanish patients with fibromyalgia. Arthritis Res Ther 2007;9:R110. [PubMed: 17961261]

24. Wichers M, Aguilera M, Kenis G, Krabbendam L, Myin-Germeys I, Jacobs N, Peeters F, Derom C, Vlietinck R, Mengelers R, Delespaul P, van Os J. The catechol-O-methyl transferase Val158Met polymorphism and experience of reward in the flow of daily life. Neuropsychopharmacology 2008;33:3030-3036. [PubMed: 17687265] 
25. Woo JM, Yoon KS, Yu BH. Catechol O-methyltransferase genetic polymorphism in panic disorder. Am J Psychiatry 2002;159:1785-1787. [PubMed: 12359690]

26. Zhang J, Quan H, Ng J, Stepanavage ME. Some statistical methods for multiple endpoints in clinical trials. Control Clin Trials 1997;18:204-221. [PubMed: 9204221]

27. Zubieta JK, Heitzeg MM, Smith YR, Bueller JA, Xu K, Xu Y, Koeppe RA, Stohler CS, Goldman

D. COMT val158met genotype affects mu-opioid neurotransmitter responses to a pain stressor. Science 2003;299:1240-1243. [PubMed: 12595695] 
Table 1

Participant Characteristics

\begin{tabular}{|c|c|c|}
\hline Characteristic & All Participants $n=89$ & Rare COMT Haplotypes Excluded $\mathrm{n}=\mathbf{8 5}$ \\
\hline Age (mean, SD) & $41(15)$ & $41(15)$ \\
\hline Female Gender (n, \%) & $53(60)$ & $50(59)$ \\
\hline \multicolumn{3}{|l|}{ COMT Genotype (n, \%) } \\
\hline Pain Vulnerable (No LPS allele) & $36(40)$ & $35(41)$ \\
\hline Not Pain Vulnerable ( $\geq 1$ LPS allele) & $53(59)$ & $50(59)$ \\
\hline \multicolumn{3}{|l|}{ Education (n, \%) } \\
\hline High School & $16(18)$ & $15(18)$ \\
\hline Some College & $12(14)$ & $12(14)$ \\
\hline College Graduate & $26(29)$ & $26(31)$ \\
\hline Post-Graduate Degree & $9(10)$ & $9(11)$ \\
\hline Other & $24(27)$ & $21(25)$ \\
\hline Missing & $2(2)$ & $2(2)$ \\
\hline \multicolumn{3}{|l|}{ Employment (n, \%) } \\
\hline Work Full Time & $52(58)$ & $51(60)$ \\
\hline Work Part Time & $18(20)$ & $15(18)$ \\
\hline No Work Outside the Home & $11(12)$ & $11(13)$ \\
\hline Student & $4(5)$ & $4(5)$ \\
\hline Disabled & $2(2)$ & $2(2)$ \\
\hline Missing & $2(2)$ & $2(2)$ \\
\hline \multicolumn{3}{|l|}{ Annual Income (n, \%) } \\
\hline Below $\$ 20,000$ & $22(25)$ & $21(25)$ \\
\hline$\$ 20,000$ to $\$ 40,000$ & $10(11)$ & $10(12)$ \\
\hline$\$ 40,000$ to $\$ 60,000$ & $23(26)$ & $22(26)$ \\
\hline$\$ 60,000$ to $\$ 80,000$ & $11(12)$ & $10(12)$ \\
\hline$>\$ 85,000$ per year & $17(19)$ & $17(20)$ \\
\hline Other/Refused & $6(7)$ & $5(6)$ \\
\hline \multicolumn{3}{|l|}{ Marital Status (n, \%) } \\
\hline Married or Living with Partner & $44(49)$ & $43(51)$ \\
\hline Single & $34(38)$ & $33(39)$ \\
\hline Divorced & $9(10)$ & $8(9)$ \\
\hline Widowed & $2(2)$ & $1(1)$ \\
\hline \multicolumn{3}{|l|}{ Injury Type (n, \%) } \\
\hline Musculoskeletal Strain Only & $80(90)$ & $77(91)$ \\
\hline Fracture ${ }^{*}$ & $4(5)$ & $4(5)$ \\
\hline Minor laceration ${ }^{* *}$ & $5(6)$ & $4(5)$ \\
\hline \multicolumn{3}{|l|}{ Neck pain severity in the ED } \\
\hline None/Mild & $44(49)$ & $40(47)$ \\
\hline Moderate/Severe & $45(51)$ & $45(53)$ \\
\hline
\end{tabular}




\begin{tabular}{|c|c|c|}
\hline Characteristic & All Participants $n=89$ & Rare COMT Haplotypes Excluded n = 85 \\
\hline AIS Severity 1 & $80(90)$ & $77(91)$ \\
\hline AIS Severity 2 & $9(10)$ & $8(9)$ \\
\hline \multicolumn{3}{|l|}{ Collision type } \\
\hline Rear-end & $40(45)$ & $38(45)$ \\
\hline Other & $49(55)$ & $47(55)$ \\
\hline Time between MVC and ED presentation (Median) & $1: 30$ & $1: 30$ \\
\hline
\end{tabular}


Table 2

Frequency of patient characteristics versus presence of moderate or severe neck pain at ED. $(n=89)$

\begin{tabular}{|c|c|c|c|}
\hline \multirow[b]{2}{*}{ Characteristics } & \multicolumn{3}{|c|}{ Moderate to Severe Neck Pain in the Emergency Department in the Hours after MVC } \\
\hline & Yes $(\%)$ & No $(\%)$ & Relative Risk $\left(95 \% \mathbf{C I}^{\mathrm{a}}\right)$ \\
\hline Age $<40$ & 57 & 44 & $1.28(.85-1.93)$ \\
\hline Female Gender & 64 & 53 & $1.24(.83-1.85)$ \\
\hline Income $<\$ 20,000$ per year & 33 & 17 & $1.67(.88-3.17)$ \\
\hline High school education or less & 29 & 16 & $1.52(.81-2.85)$ \\
\hline Car drivable after MVC & 67 & 67 & $1.00(.61-1.62)$ \\
\hline Rear-ended & 50 & 38 & $1.28(.83-1.96)$ \\
\hline Airbags Deployed & 24 & 34 & $.79(.52-1.20)$ \\
\hline COMT pain vulnerable genotype ${ }^{*}$ & 76 & 41 & $2.11(1.33-3.37)$ \\
\hline
\end{tabular}

* Defined as COMT genotype which does not contain one or more "low pain sensitivity"7 haplotypes 
Table 3

Final stepwise logistic regression models $(\mathrm{p}$ in $=.1, \mathrm{p}$ out $=.15$ ) assessing predictors of emergency department somatic symptoms after motor vehicle collision.

\begin{tabular}{llrrc}
\hline Dependent Variable & Independent Variable & Exp $(\boldsymbol{\beta})$ & Wald & p \\
\hline Moderate or Severe & Income & .728 & 3.058 & .080 \\
Neck Pain (Yes/No) & COMT Pain Vulnerable Genotype & 3.326 & 5.359 & .021 \\
& Constant & 1.889 & 1.046 & .306 \\
Moderate or Severe & COMT Pain Vulnerable Genotype & 2.667 & 4.146 & .042 \\
Headache (yes/no) & Constant & .577 & 2.878 & .090 \\
Moderate or Severe & COMT Pain Vulnerable Genotype & 4.222 & 3.942 & .047 \\
Dizziness (yes/no) & Constant & .079 & 17.924 & $<.0001$ \\
\hline
\end{tabular}

* Candidate predictors for each model: demographic characteristics (age, gender, income, education), crash and injury characteristics (highest AIS score, car drivable at scene (yes/no), airbags deployed (yes/no)), and presence or absence of COMT pain vulnerable genotype. 
Table 4

Final stepwise linear regression models $(\mathrm{p}$ in $=.1$, $\mathrm{p}$ out $=.15$ ) assessing predictors of emergency department psychological symptoms after motor vehicle collision.

\begin{tabular}{|c|c|c|c|c|}
\hline Dependent Variable & Independent Variable ${ }^{*}$ & $\beta$ & $\mathbf{t}$ & $\mathbf{p}$ \\
\hline \multirow[t]{3}{*}{ Dissociative symptoms ${ }^{\dagger}$} & COMT Pain Vulnerable Genotype & -.264 & -2.317 & .023 \\
\hline & Rearend Collision & -.202 & -1.774 & .080 \\
\hline & Constant & & 14.140 & $<.0001$ \\
\hline \multirow[t]{3}{*}{ Distress symptoms ${ }^{¥}$} & Income & -.278 & -2.388 & .020 \\
\hline & Airbag Deployed (Yes/No) & .234 & 2.003 & .049 \\
\hline & Constant & & 9.751 & $<.0001$ \\
\hline
\end{tabular}

